\title{
Reconsidering harm in psychiatric manuals within an explicationist framework
}

\author{
Mia Biturajac $^{1}\left[\right.$ Marko Jurjako $^{1}[$
}

Accepted: 11 December 2021 / Published online: 17 January 2022

(c) The Author(s), under exclusive licence to Springer Nature B.V. 2021

\begin{abstract}
The notion of harm has been a recurring and a significant notion in the characterization of mental disorder. It is present in eminent diagnostic manuals such as DSM and ICD, as well as in the discussion on mental disorders in philosophy of psychiatry. Recent demotion of harm in the definition of mental disorders in DSM-5 shows a general trend towards reducing the significance of harm when thinking about the nature of mental disorders. In this paper, we defend the relevance of the notion of harm in the characterization of mental disorder against some of these attacks. We approach this issue by using the method of conceptual explication pioneered by Rudolf Carnap. Within this framework, we argue that keeping the notion of harm not only helps to discriminate what is pathological from the nonpathological but also prevents potential misuses of psychiatric authority.
\end{abstract}

Keywords DSM-5 $\cdot$ Explication $\cdot$ Harm $\cdot$ Dysfunction $\cdot$ Mental disorder

\section{Introduction}

The place of the notion of harm within manuals such as the Diagnostic Statistical Manual of Mental disorders (DSM5, American Psychiatric Association 2013) and the International Classification of Diseases (ICD-10, World Health Organization 1992) is controversial. For instance, in DSMIV harm was considered a necessary condition for something to be a mental disorder, while in DSM-5 harm is something that only usually and not necessarily accompanies behavioral, psychological or biological dysfunctions underpinning mental disorders (Amoretti and Lalumera 2019; Cooper 2013). Some authors are reluctant to accept this evolution of DSM's definition of mental disorder (e.g. Cooper 2013;

Both authors contributed equally to the paper and should be considered as co-first authors.

Marko Jurjako

mjurjako@ffri.uniri.hr

Mia Biturajac

mbiturajac@ffri.uniri.hr

1 Department of Philosophy, Faculty of Humanities and Social Sciences in Rijeka, University of Rijeka, Sveučilišna Avenija 4, Rijeka, Croatia
Telles-Correia 2018), while others provide arguments to support it (Amoretti and Lalumera 2019).

In this paper we will focus on a recent discussion by Cristina Amoretti and Elisabetta Lalumera (2019) in which they argue that the demotion of harm in the general definition of mental disorder in DSM-5 is justified. They argue that the notion of harm is too vague or unclear and thus should be discarded from the definition of the concept of mental disorder. Moreover, they claim that regardless of the role that harm historically played in thinking about mental disorder, it can be replaced by a notion of dysfunction. To evaluate their arguments we set out to make explicit the theoretical and practical requirements that a concept of mental disorder should satisfy given the roles that it plays in social practices where diagnostic manuals such as DSM are used (Lemoine 2013; Schwartz 2014; Walker and Rogers 2018; Powell and Scarffe 2019). In this regard, we think that progress in addressing the role of harm can be made by adopting the explicationist methodology (see, also Schwartz 2014; Griffiths and Matthewson 2018). Philosophical explication involves actively devising a concept with respect to theoretical and practical concerns that regulate and set goals for our conceptual projects (Carnap 1971; Dutilh Novaes 2018; Dutilh Novaes and Reck 2017; Brun 2016). This methodology provides criteria of adequacy that enable the integration and balancing of the theoretical and ethical considerations 
that should guide concept formation in particular domains of inquiry (e.g. Dutilh Novaes 2018).

Based on this methodology we will argue that the recent arguments advanced by Amoretti and Lalumera that harm should not be included as a necessary condition in the definition of mental disorder in DSM-5 are not compelling. Within the explicationist perspective, their reasons for claiming this might be supported by the requirements of exactness and simplicity. We will argue that what might be gained in exactness or simplicity by discarding the notion of harm would be lost in theoretical and ethical fruitfulness exhibited by a notion of mental disorder that involves harm and dysfunction as separate components.

We want to emphasize that the context and the scope of this paper is limited to the view of disorder found in diagnostic manuals such as DSM, which is also the context in which Amoretti and Lalumera's (2019) arguments against harm are situated. Within this context, we will argue that an adequate notion of mental disorder, beside already entrenched notion of dysfunction, should include an explicit reference to harm. As will be explained later in the text, we see the role of the dysfunction component as capturing the descriptive considerations that ground psychiatric nosology, while the role of the harm component is to capture human values and perspectives, i.e. normative considerations that shape psychiatric nosology and practice. We recognize that there might be other two-partite views in the general debate on how to define the concept of mental disorder, or more generally health and disease, that do not specifically involve the notion of dysfunction or harm (for discussion, see Murphy 2006, ch. 2; see, also Ereshefsky 2009; Stegenga 2015). However, discussing these views would fall outside of the scope and the aims of this paper.

The rest of the paper is structured as follows. In Sect. 2, we document the changes that characterize conceptualizations of the notion of mental disorder from DSM-IV to DSM-5. In this regard, we note the demotion of the harm component from being a necessary condition that characterizes mental disorders to something that is not necessarily associated with them. In Sect. 3, we introduce the explicationist methodology, as elaborated by Rudolf Carnap (1971) and contemporary commentators. In Sect. 4, based on this methodology, we argue that adopting an account of mental disorder that involves harm and dysfunction as separate components can be justified. Moreover, we discuss and evaluate recent arguments provided by Amoretti and Lalumera (2019) against using harm to define mental disorders in the context of DSM-5. In Sect. 5, we provide further reasons for thinking that the explicationist requirement of fruitfulness favors a view of mental disorder that provides separate criteria for the application of the harm and dysfunction components.

\section{The concept of mental disorder in DSM: between naturalism and normativism}

The general philosophical debate on the notion of mental disorder involves a continuum of positions between naturalists and normativists (Bolton 2008). Naturalists view mental illness as reducible to their biological characteristics and think that mental disorders can be accounted for in biological terms, often conceptualized as some kind of biological dysfunction (e.g. Boorse 1975; 2014; Hausman 2012; Schwartz 2007; for a recent overview, see Veit 2021). Thus, they can be characterized as dysfunction-only views of mental disorder. On the other side of the spectrum are normativists according to which mental disorders are essentially value-laden, i.e. they can be defined by using value concepts such as harm, rational agency, treatability, unluckiness, and so on (e.g. Fulford 1989; Nordenfelt 1995; Cooper 2002; Bolton 2008). For instance, Rachel Cooper (2002) considers disorders to be conditions that are bad things to have, which we are unlucky to have and which are potentially medically treatable. In between are the so-called hybrid theorists who try to accommodate the insights of both normativists and naturalists (Glover 1970, ch. 6; Wakefield 1992; see, also Stegenga 2015; Powell and Scarffe 2019). On such accounts the notion of mental disorder contains two components, one descriptive, anchored in scientific findings, usually realized through the notion of dysfunction, and one normativist, in the form of harm (see, e.g. Wakefield 1992; Murphy 2006).

Parallel to, and somewhat independent of the philosophical debate, various stakeholders participating in discussions on the development of psychiatric diagnostic manuals have also debated what would be an appropriate definition of the concept of mental disorder. In this regard, it is important to note that the American Psychiatric Association in the third edition of the Diagnostic Statistical Manual (DSM-III) adopted a kind of hybrid view of mental disorders (see, e.g. Spitzer 1973; 1981). ${ }^{1}$ This view was dominant until DSMIV, where the concept of mental disorder was defined as a

a clinically significant behavioral or psychological syndrome (...) that is associated with present distress (e.g., a painful symptom) or disability (i.e., impairment in one or more important areas of functioning) or with a significantly increased risk of suffering death, pain, disability, or an important loss of freedom. (...)

\footnotetext{
${ }^{1}$ It should be noted that by "hybrid" we refer to accounts that keep separate the harm and dysfunction components when defining the notion of mental disorder, as it has been the case with DSM-IV. In this regard, hybrid accounts of mental disorder should not be confused with Wakefield's harmful dysfunction account that presents one instance of hybrid accounts where the dysfunction component has a specific evolutionary-etiological interpretation. See also Footnote 2.
} 
Whatever its original cause, it must currently be considered a manifestation of a behavioral, psychological, or biological dysfunction in the individual. (DSM-IV, APA 1994, xxi-xxii)

Here the notion of mental disorder is defined in terms of two separable components involving harm and dysfunction. The criteria for assessing whether a behavioral, psychological, or biological dysfunction is present are not further specified besides emphasizing that the dysfunction should reside within an individual. ${ }^{2}$ The harm component is in DSM conceptualized as involving things such as personal distress, disability as impairment in one or more important areas of everyday functioning, and increased risk of suffering death, pain, disability, or loss of freedom. For the purposes of this paper we will adopt this DSM notion of harm that broadly encompasses considerations of well-being and the ability to function normally as judged by the standards of the western culture within which DSM was created (Cooper 2005). In addition, this notion allows that social harms can also be part of the criteria for ascribing the label of mental disorder. For instance, this perspective is captured in DSM-5 as follows:

Socially deviant behavior (e.g. political, religious, or sexual) and conflicts that are primarily between the individual and society are not mental disorders unless the deviance or conflict results from a dysfunction in the individual (...). (DSM-5, APA 2013, p. 20)

This indicates that conditions primarily defined in terms of social deviance and harm such as those involving antisocial personalities can be considered as mental disorders only if they are underpinned by some form of internal dysfunction (Jurjako et al. 2021).

\footnotetext{
${ }^{2}$ In philosophy of psychiatry there are several views about the nature of the relevant behavioral, psychological, and biological capacities and the normative standards that determine when they are dysfunctional. Biologically oriented accounts define these capacities in terms of evolved biological or psychological traits whose proper function is determined by natural selection (see, e.g. Wakefield 1992; Griffiths and Matthewson 2018) or how well they contribute to biologically relevant goals that pertain to reproduction and/or survival (see, e.g. Boorse 1977; Garson and Piccinini 2013). Non-biologically oriented accounts tend to delineate the relevant psychological capacities and their standards of functioning by considering the preconditions for being a rational agent capable of accomplishing ethically valued goals in life (see, e.g. Graham 2013; for discussion, see Varga 2017). For the purposes of this paper, we do not have to take a stance on the right account of the relevant capacities and their standards of proper function. This issue seems to be orthogonal to our argument that the change in the notion of mental disorder from DSM-IV to DSM-5 is not favored by an explicationist analysis and that an adequate explicatum of the concept of mental disorder within this context should include an explicit reference to harm. Thanks to an anonymous reviewer for pressing us to be more explicit on this issue.
}

Importantly, the conceptual shift in the DSM-5 occurred because the harm condition was demoted from a necessary condition characterizing mental disorders to something that is "usually associated with" them (Cooper 2013; Telles-Correia 2018). Mental disorder is currently conceptualized as "a dysfunction in the psychological, biological, or developmental processes (...) [that] are usually associated with significant distress or disability in social, occupational, or other important activities" (APA, 2013, p. 20). The direction of this shift goes towards dysfunction-only views because it allows mental disorders to be individuated solely in terms of biological, psychological, or behavioral dysfunction without necessarily being associated with some form of harm. The question we want to address is whether this shift towards dysfunction-only views is justified.

Amoretti and Lalumera (2019) provide theoretical and practical reasons for thinking that this shift in conceptualization of mental disorders is justified. We will evaluate their reasons within the explicationist framework. We will argue that although Amoretti and Lalumera offer valid pro tanto reasons for thinking that this shift is justified, still there are weightier reasons for retaining a hybrid view of mental disorder that make explicit the harm component. Before discussing their arguments, in the next section we will introduce and explain why it is a good idea to use the explicationist framework, and delineate some of the roles that the concept of mental disorder plays within DSM and social practices that should guide our explication of it.

\section{Explication, criteria of adequacy, and the concept of mental disorder}

Our task is to determine what would be an appropriate concept of mental disorder for diagnostic manuals such as DSM5. Accordingly, our context of inquiry is defined by the roles that DSM and similar diagnostic manuals play in psychiatry. In particular, categorizing mental disorders as in the DSM should enable reliable diagnoses, communication between researchers, epidemiological studies, and devising appropriate treatments (Cooper 2005; Beebee and SabbartonLeary 2010). Their primary role in research and treatment is grounded in the fact that mental disorders usually denote conditions that cause distress and pain to a person, reduce their functional abilities, and in other respects negatively affect different aspects of their life. Moreover, these uses of DSM are extended to practical contexts such as granting different rights or exempts from obligations to a person. For instance, if a person is diagnosed with a disorder included in DSM, this might provide grounds for absolving them of their work duties; or we might hold them less responsible if they performed an immoral or criminal act; or we might think that they should receive some sort of financial compensation 
for their pain or an opportunity to get well, and so on (see Cooper 2005).

We think that in devising the definition of mental disorder appropriate for DSM, we should always keep one eye on the aforementioned considerations and aims with which it is used. The notion of mental disorder, as well as the DSM, does not operate in a vacuum but is entrenched in social practices and relates to various stakeholders. To remain cognizant of these considerations we rely on the explicationist framework that has recently gained traction in philosophy of psychiatry (Schwartz 2014; Matthewson and Griffiths 2017; Jurjako and Malatesti 2020; see, also Amoretti and Lalumera 2021, p. 12). What recommends this framework is that it makes perspicuous how the relevant theoretical and practical considerations can be upheld and balanced when devising a concept appropriate for a particular domain of inquiry. Let us consider in more detail what the process of explication involves.

According to Carnap " $[\mathrm{t}] \mathrm{he}$ task of explication consists in transforming a given more or less inexact concept into an exact one or, rather, in replacing the first by the second" (Carnap 1971, 3, emphasis in the original). In other words, explication is the process by which we take a concept that is inexact in problematic ways, and we make it more exact according to our needs. The concept we start with is called explicandum, while the product of explication is called explicatum. The explicandum may be a term coming from everyday or scientific language that we want to make more precise. The explicatum then is the product of explication. Given that the task of explication is conceptual transformation, explicatum can only be judged as more or less adequate in comparison to other proposed explicata (Carnap 1971, p. 4).

Carnap provides the following criteria for judging the adequacy of an explicatum: (1) similarity to the explicandum; (2) exactness; (3) fruitfulness; and (4) simplicity. These criteria can be weighted differently depending on the context of inquiry (Dutilh Novaes and Reck 2017). For instance, it is important that the explicatum be somewhat similar to the meaning of the explicandum because otherwise we run the risk of simply changing the topic. Simplicity seems to be the least important. Carnap indicates that when we have two or more proposed explications of a concept and one is simpler than the others, while in terms of other criteria they are the same, then we might prefer the simpler one. Arguably the most important criteria for Carnap are exactness and fruitfulness, and where the two conflict fruitfulness might be the requirement carrying the most weight (Dutilh Novaes and Reck 2017). Fruitfulness for Carnap means allowing the formulation of empirical generalizations or theorems in the case of logic and mathematics. However, as we will see below, in recent literature fruitfulness has been explicitly given a more encompassing role, which enables us to devise concepts that beside explanatory can satisfy practical and ethical desiderata of our intellectual inquiries (see Dutilh Novaes 2018; Carus 2009).

In general, the satisfactoriness of explication should be judged relative to a domain of inquiry we find worthwhile pursuing (Dutilh Novaes 2018). In this regard, the requirement of fruitfulness plays the most important role. Carnap explains it with a reference to the concept FISH. In common language, we might be inclined to characterize as fish all creatures living in water that have similar morphological features. To make it more precise and scientifically fruitful, we can replace it with the concept that Carnap labels "piscis". The concept PISCIS denotes cold-blooded vertebrates that live under water. PISCIS is more fruitful than FISH because it delineates a more specific class of animals, thereby allowing us to formulate more empirical generalizations (such as PISCIS typically have gills and reproduce by spawning eggs), which in turn can ground reliable inferences and be usefully applied in other ways.

In addition to theoretical or empirical generalizations, fruitfulness can be evaluated with respect to our practical and ethical concerns that aim at enhancing the human condition or even correcting and protecting human rights (Carus 2009). For instance, Catharina Dutilh Novaes (2018) compares explication to the project of amelioration as expounded by Sally Haslanger (2012), where the goal is to engineer concepts in a way that can expose social injustices and in turn be used to correct them by reforming the relevant social practices. ${ }^{3}$ This aspect of explication is important for the present context because, as mentioned earlier, the notion of mental disorder provides the foundation for DSM categories, which in turn play important roles across various social practices, spanning from medicine, development of

\footnotetext{
${ }^{3}$ Haslanger is most well-known for applying the ameliorative analysis to concepts of gender and race. The project of conceptual amelioration first starts with critical examination of the purposes and practices in which a concept is used. For instance, in the case of race and gender the problems we want to address are the social inequalities, lack of access to public goods, systemic discrimination, stigmatization, etc. that are based on unjust social practices and unequal power constellations between various groups of people. In the second step, ameliorative analysis suggests devising a concept that would serve the purposes of exposing and then reducing these social injustices. Haslanger suggests that in the case of race the following hierarchical concept would serve this purpose: "A group is racialized iff $f_{\mathrm{df}}$ its members are socially positioned as subordinate or privileged along some dimension (economic, political, legal, social, etc.), and the group is "marked" as a target for this treatment by observed or imagined bodily features presumed to be evidence of ancestral links to a certain geographical region" (Haslanger 2012, p. 236). Philosophical explication is similar to ameliorative analysis to the extent it takes into consideration the goals and purposes of an inquiry (including political and ethical ideals) with the aim of devising a concept that can satisfy them. For a detailed comparison of the two approaches to conceptual engineering, see Dutilh Novaes (2018, pp. 1025-1028).
} 
treatments, social health, and insurance policies, to criminal law (e.g., the insanity defense) (Cooper 2005). Thus, in general, discussing things within the explicationist framework should enable us to sharpen our focus on the desiderata that a suitable notion of mental disorder should fulfil and provide criteria for balancing them. In the next section, we will examine through the explicationist lens whether a dysfunction-only or a hybrid explication of the concept of mental disorder better captures the theoretical and practical roles that DSM mental disorders play in psychiatry and associated social practices.

\section{Does an explicationist framework support a dysfunction-only view of mental disorders?}

We start with reasons why a good explicatum of the concept of mental disorder would favor a DSM-IV type of hybrid account. Then we examine Amoretti and Lalumera's (2019) arguments that a DSM appropriate notion of mental disorder needs only to rely on the notion of dysfunction.

It might be argued that exactness and fruitfulness requirements count in favor of retaining the hybrid two-component account of mental disorder as formulated in DSM-IV. Given the mentioned roles that the concept of mental disorder plays in our psychiatric and associated social practices, the hybrid account might seem to contend a more exact concept. It allows us to explicate underpinnings of mental disorders in terms of dysfunctional or impaired internal biological or psychological mechanisms, which are descriptive and amenable to scientific research. Moreover, the harm component captures in a more precise way the idea that mental disorders negatively affect people's lives and makes clear the normative grounds for justifying treatments and granting special rights and protections to those suffering from mental disorders.

This view also fulfills the criterion of fruitfulness because it provides a bridge between theoretical research-oriented aspects of psychiatry with its inherently practical applications in alleviating problems that people experience and cannot cope with on their own (see, also Murphy 2006, p. 19 , on the two stage view of psychiatry). On the one hand, the dysfunction component connects the notion of mental disorder with scientific investigations of normal and abnormal functioning of biological and psychological mechanisms allowing us to formulate empirical generalizations, hypotheses, and perform scientific studies (see Griffiths and Matthewson 2018). On the other hand, having explicit reference to harm enables us to uncover and explicitly reflect on the connections between the scientific studies of the causal basis of mental disorders with ethical and social practices that involve thinking about how harms should be reduced, avoided, and fairly compensated (see, also Powell and Scarffe 2019). For instance, when thinking about how to direct resources for research and devise psychiatric treatment polices our judgments are typically guided by categories of mental disorders as adopted by diagnostic manuals (Cooper 2005; Jurjako and Malatesti 2020; Gagné-Julien 2021). Thus, to make ethically justified decisions when creating the relevant psychiatric categories and when thinking how to invest resources and which conditions to treat, we should be sensitive to the relevant normative considerations that go beyond thinking about whether some condition is dysfunctional or not (Stegenga 2021).

Amoretti and Lalumera (2019), however, offer reasons for thinking that the concept of mental disorder would be clearer and more exact without the harm component. They indicate that harm in diagnostic manuals is typically conceived as distress or disability, the latter of which involves impairment in important areas of functioning. The main problem they indicate for construing harm as distress is that the concept of distress is ineliminably vague, or we might say inexact, and thus in some cases inappropriate for deciding whether a condition should be introduced to psychiatric nosologies (see Amoretti and Lalumera 2019, pp. 326-27). For instance, they claim that there are no clear boundaries between adaptive and pathological distress. Distress might be adaptive, and therefore not a symptom of illness when it is an appropriate response to stressful life events, such as being extremely sad for the loss of a loved one (Phillips 2009). Another reason they offer against harm as distress is that a person might not have sufficient insight into their condition. For instance, hoarding might not be directly distressful to a person, nonetheless it can cause them problems in everyday functioning and social relations (Amoretti and Lalumera 2019, p. 327). Thus, thinking of harm as distress might lead to many instances of false negatives, where we might treat as healthy those people who in fact need psychiatric attention.

However, explicating the notion of disorder solely in terms of dysfunctions would not remove all vagueness from the concept of mental disorder. In fact, because of ineliminable variation in biological and psychological traits it is expected that there will be some borderline cases for which it will not be clear whether they should be considered as dysfunctional or just a healthy variation (Griffiths and Matthewson 2018). In addition, the aforementioned problem of false negatives does not seem to do better on dysfunctiononly conceptions of mental disorder. Hybrid accounts make salient the fact that often a condition being just dysfunctional or abnormal does not necessitate psychiatric or medi$\mathrm{cal}$ attention. For instance, delusional experiences seem to be dysfunctional or abnormal, but not all cases of delusional experiences are distressful or in other ways harmful (see, e.g. Lancellotta and Bortolotti 2020). Thus, when a person 
has delusional beliefs "under control" it seems reasonable to think that they do not need psychiatric attention. However, by parity of reasoning it seems that when they do need psychiatric attention it is because they are somehow harmed by delusional experiences and beliefs.

These considerations indicate that the supposed vagueness of the notion of distress and its potentially negative consequences for psychiatric practice seem to be overstated. At most, they show that harm cannot be reduced to distress. Indeed, as acknowledged by Amoretti and Lalumera (2019), conditions are harmful when they negatively affect a person's well-being and experiencing distress is just one way in which a person could be harmed.

As noted earlier, in psychiatric manuals this other aspect of harm is construed as a lack of ability to function in everyday situations. However, Amoretti and Lalumera maintain that due to conceptual clarity even on this more expanded interpretation of harm we should keep the notions of mental disorder and harm separated. They indicate that disability is commonly construed as context-dependent. For instance, a person in a wheelchair is disabled in an environment where they cannot normally function due to physical and other limitations. However, in an environment where there are ramps, elevators, and everything is accessible, the person in a wheelchair might not be disabled from normal everyday functioning. Amoretti and Lalumera claim that separating mental disorders from harm as disability achieves conceptual clarity because it

helps in identifying cases where proper environmental changes and provision of social resources, and not, say, individual therapy or medications, would make a difference to patients' conditions. (Amoretti and Lalumera 2019, p. 329)

Here they make a point that mental disorders are typically conceived as those things that cannot be easily treated by changing the environment, rather they are related to internal dysfunctions that we tend to treat by medicalizing them (see, also Cooper 2020, on the location problem). We agree with this consideration. Nevertheless, we think that removing harm as a criterion for thinking about psychiatric nosology will not help with achieving clarity in this respect. Harm primarily plays the role in determining which internal dysfunctions we should treat as pathological. Without the harm component, we will have tough time deciding what conditions should be introduced into psychiatric nosologies, on which conditions we should spend resources in investigating and developing therapies, and finally which conditions we should treat whether by medical or some other means (Stegenga 2021). Thus, the alleged downsides of having the harm criterion seem to be outweighed by the general considerations that without thinking about which dysfunctional conditions are actually harmful to people, we would be oblivious to the practical constraints within which psychiatry as well as the rest of the medicine functions (see, also Gagné-Julien 2021).

Amoretti and Lalumera seem to disagree. They claim that discarding harm will bring practical, political, and ethical benefits in the form of bringing psychiatry closer to somatic medicine where, according to them, disorders are not necessarily viewed as harmful. In fact, they claim that having the harm component in somatic medicine might be detrimental:

Consider asymptomatic early-stage cancers, which cause neither distress nor disability - given the benefits of early diagnosis in terms of prognosis, imposing a harm condition would amount to preventing the possibility of treating and saving many patients. Similar considerations can easily be extended to mental disorders as well. (Amoretti and Lalumera 2019, p. 330)

These considerations are not persuasive. Clearly, we medicalize cancers and other types of dysfunctional conditions and processes because we think they are harmful, in the sense of well-being reducing. If we did not think that early stages of cancer eventually lead to harm and death, we would not be interested in treating them or thinking of them as disorders. The same holds for mental disorders. An evaluative component related to harm is needed to capture the normative roles that the notion of mental disorder as used in diagnostic manuals plays in psychiatric nosology and its downstream effects in terms of investing resources in finding treatments.

Moreover, it is spurious that a dysfunction-only conceptualization of mental disorders would be more similar to conceptualizations of disorder as used in general medicine. In particular, the Covid pandemic made everyone aware of the importance of the distinction between a medical disorder and its underlying cause (see, e.g. Kennedy and Cwik 2021). Covid-19 involves symptoms such as problems with the respiratory system, fever, anosmia, and tiredness, which are caused by the virus Sars-CoV-2. However, people infected with Sars-CoV-2 who do not have the symptoms of Covid19 are not considered to be ill or suffering from Covid-19 exactly because the virus does not harm them (see, also Wakefield 2014). Thus, if we want to get psychiatry closer to somatic medicine it seems more fruitful to explicitly acknowledge the important roles that harm plays in conceptualizing medical disorders.

However, even if it is granted that a dysfunction-only view of mental disorder provides a more exact and simpler notion, we will argue that adopting a hybrid two-component account would provide a more fruitful concept of mental disorder. As mentioned earlier, not all requirements for explication bear equal weight (Dutilh Novaes and Reck 2017; Dutilh Novaes 2018; Brun 2016). In line with this, we think that fruitfulness of hybrid accounts outweighs the potential benefits of having a more simplified, or even exact, notion of 
mental disorder without the explicit normative component. In what follows we provide reasons for thinking this.

\section{The ethical and theoretical fruitfulness of hybrid accounts of mental disorder}

The fruitfulness of the hybrid accounts that we want to highlight has to do with the practical and ethical values that frame the goals of explication in the present context. More specifically, we argue that incorporating harm, among other things, satisfies the ethical requirements that include protecting personal freedoms against the potential misuses of psychiatry, and capturing the perspective of the people whom we contemplate labelling as mentally disordered. Both of these points can be appreciated by reminding ourselves how harm was eventually introduced in DSM-III.

In 1973, homosexuality was removed from the DSM. This move had a significant impact on the recognition of gay rights throughout the globe. A center figure in this endeavor was Robert Spitzer (see, e.g. Spitzer and Williams 1982). He recognized that uncontroversial cases of mental disorders involved some form of personal distress on the part of a suffering individual. Accordingly, Spitzer concluded that persons diagnosed with mental disorders, besides exhibiting some sort of social, psychological, or biological dysfunction, must experience distress or disability. Since homosexuality is not intrinsically distressing, and given that gay people can be happy, otherwise healthy and high functioning, it was concluded that homosexuality is not a disorder (Spitzer 1973). As a result, harm has been included in the DSM-III as one of the essential criteria of mental disorders.

The idea that mental disorders involve harm in terms of distress or problems in everyday functioning, can be seen as a rational reconstruction sensitive to the relevant moral considerations, that members of the American Psychiatric Association used to remove homosexuality from their list of disorders. Importantly, these changes would not have happened if it were not for the Gay rights movement and activists who argued that homosexuality should be considered a normal variation in the human population (Bayer 1987; Drescher 2015). They brought to the attention of the professional public, via public events, educational panels, and even protests that treating homosexuality as a disorder has illegitimate stigmatizing effects, when, in fact, gay people normally do not suffer from psychological disturbances.

In this regard, we maintain that introducing harm as a necessary component in the definition of mental disorders served to introduce the perspectives of agents and groups whose psychiatric status was under scrutiny. Therefore, by keeping the harm component in the definition of mental disorder as exhibited in DSM-IV we reduce the opportunity for different social forces to use psychiatry for exerting power over individuals simply for being considered as social deviants, misfits, and non-conformists in various respects. ${ }^{4}$

In spite of this, it might be insisted that the notion of dysfunction alone can provide an adequate explication of the concept of mental disorder. In particular, Amoretti and Lalumera claim that regardless of the role that harm played in the history of psychiatry, it is now superfluous because it can be accommodated by the notion of dysfunction. Accordingly, they maintain:

that the harm requirement is now no longer necessary in order to exclude homosexuality from psychiatric diagnoses, as the other criterion for classification as a mental disorder - the dysfunction requirement—can provide sufficient reason for such exclusion in its own right. Current theories of homosexuality largely agree that it is a case of normal variation, with no dysfunction in play. (Amoretti and Lalumera 2019, p. 324)

Now, it is not entirely clear why Amoretti and Lalumera think that the notion of dysfunction can replace the role that harm played in removing homosexuality from the list of disorders. Especially because, even if we agree that there is a general consensus that homosexuality is not associated with dysfunctions (cf. Cooper 2020, p. 145), it seems clear that this consensus would not be achieved if it were not for the Gay rights activists arguing that it is not harmful (Drescher 2015).

One way to interpret this is the idea that the notion of dysfunction is already value-laden in relevant respects. Amoretti and Lalumera, among others, recognize that the notion of dysfunction will need to make room for some normative considerations (Amoretti and Lalumera 2021; Cooper 2020; Kingma 2014; Schwartz 2007). In particular, the line-drawing problem makes salient the difficulty of providing a completely objective notion of dysfunction (see, e.g. Schwartz 2007; Kingma 2014; Rogers and Walker 2017; Cooper 2020; Schramme 2021). To illustrate, Wendy Rogers and Mary Walker indicate that "hypertension [...] is defined as blood pressure above 140/90 mmHg" (Rogers and Walker 2017, 410), which is a boundary that does not strictly delineate function from dysfunction but is set somewhat to tease apart those at higher risk of coronary disease from those without

\footnotetext{
${ }^{4}$ The justification that we frame here in terms of practical/ethical fruitfulness, can also be cast in terms of responsible conceptual engineering that is sensitive to the pragmatic genealogy of the conceptual practice underlying the concept of mental disorder (see Queloz 2021, ch. 2). Our argument can be construed as saying that the historical removal of homosexuality from DSM-III and the needs for personal and social justice which this was a response to provide a vindication of our claim that a theoretically and ethically justified way of constructing the concept of mental disorder should involve harm.
} 
pathological potential. ${ }^{5}$ This indicates that in determining whether a condition is a dysfunction, the cut-off point will be drawn somewhat arbitrarily according to other considerations not necessarily intrinsic to the dysfunction properties, for example those indicating whether certain levels of functioning lead to harmful consequences, how easily and effectively some condition is treatable, and so on.

From these types of considerations, we could interpret Amoretti and Lalumera to claim that the dysfunction-only notion of disorder is weakly normative. They introduce the distinction between strong and weak normativism in Amoretti and Lalumera (2021). Weak normativism signifies that "no evaluative concept explicitly figures as a component of the definition of disease, but some value-laden concepts or judgments may intervene in the operationalization of some of such components" (Amoretti and Lalumera 2021 , p. 8). Conversely, according to strong normativism, evaluative concepts, such as badness, harm, disability, and so on, explicitly figure as components of the definition of disease. Amoretti and Lalumera point out that many scientific concepts are weakly normative. For instance, they consider the concept of confidence interval that is used to estimate a value of an unknown parameter from observed data. Regarding this concept they indicate that it

can be seen as expressing the range of false positives and false negatives (...). When false negatives are evaluated as more dangerous than false positives-for example, when there is a relevant public health risk-a larger confidence interval is tolerated and can even be intentionally chosen. This can be the case, for example, of assessing the correlation between heavy metals in drinking water and severe headaches. Differently, smaller confidence intervals are chosen when false positives are to be avoided, as when testing whether, say, a vitamin $\mathrm{C}$ supplement is protective against the common cold. (Amoretti and Lalumera 2021, p. 10)

Thus, the notion of confidence interval is weakly normative because our values can play a role in its application conditions, but they do not figure in the definition of the concept. However, if the notion of dysfunction is weakly normative in this sense, then we do not think it can capture the roles that we argued harm plays and should play in the

\footnotetext{
5 Strictly speaking, hypertension is a risk-factor indicating that a heart might be prone to malfunction. Put this way, it is not immediately clear how the line-drawing problem in risk-factors translates to a line-drawing problem for dysfunctions (for discussion, see Hofmann 2021; Schramme 2021). However, we will bracket this issue, because our point is that even if the line-drawing problem shows that dysfunction-requiring accounts cannot provide a completely objective account of dysfunction, still this does not favor dysfunction-only accounts to two-component hybrid accounts of mental disorder.
}

notion of mental disorder. This is because it only makes harm contingently important for ascribing mental disorder and consequently building psychiatric nosologies. However, as argued earlier, the historical reasons that played a role in removing homosexuality from earlier versions of DSM indicate that the role of harm pertains, among other things, to recognizing the perspectives of people affected by the psychiatric practice. Thus, if this role of harm is not necessarily recognized in ascriptions of dysfunctions, then we maintain that the requirement of ethical fruitfulness would disfavor dysfunction-only accounts of mental disorder.

This problem might be remedied if we adopt a strong normative reading of dysfunction and allow that harm plays a necessary role in ascribing dysfunctions (for discussion, see Hofmann 2021). However, this does not seem to be compatible with Amoretti and Lalumera's (2019) argument. To recall, they argue that removing harm from the definition of the concept of mental disorder in DSM was a good thing. So, to accept harm as a necessary component of dysfunction would undermine their original reasons for defending a dysfunction-only view of mental disorder.

Furthermore, there are other reasons for not conflating the roles of dysfunction and harm when explicating the notion of disorder. As we have argued earlier the notion of dysfunction, on the one hand, connects psychiatry to other scientific domains where it plays explanatory roles and provides grounds for inferences, diagnosis, devising treatments, and so on. On the other hand, the harm component connects psychiatry to the lived human experiences and directs our attention to those dysfunctional conditions that can be investigated and treated by medical means. In this regard, as we explain below, even accepting that the notion of (dys)function is value-laden, theoretical and ethical fruitfulness would favor adopting a form of hybrid view that keeps distinct the more scientific dysfunction component and person-oriented harm component.

First, theoretical fruitfulness directs us towards adopting an account of dysfunction that could apply across different disciplines. As John Matthewson and Paul Griffiths clearly indicate "notions of normality, abnormality, pathology, and physiology are essential to understanding the biology of living things, even in cases where human values play no role whatsoever" (Matthewson and Griffiths 2017, p. 464; cited in Veit 2021, p. 295; see, also Griffiths and Matthewson 2018). Accordingly, when assessing whether an animal suffers from a disease a proper notion of biological function is usually presupposed that does not rely on human conceptions of harm or well-being. In this regard, adopting a notion of dysfunction that necessarily relies on human conception of harm would sever the notion of dysfunction in the human case from the notion of dysfunction as used across biological sciences (Veit 2021). Given that humans belong to the natural world theoretical fruitfulness plausibly requires a 
notion of function/dysfunction that would enable making generalizations and reliable inferences that can apply across biological species (for discussion, see Griffiths and Matthewson 2018).

Second, we maintain that ethical fruitfulness would favor not conflating the roles that dysfunction and harm play in the notion of mental disorder. In fact, we maintain that such accounts provide safeguarding, meaning that they can be enforced for securing the society against the potential misuses of psychiatry while at the same time provide more conceptual and practical resources for people to ask for recognition and acceptance (Powell and Scarffe 2019). Historic examples of abuses of psychiatric/medical authority abound with conditions such as drapetomania ('disorder' of slaves running away from their owners), hysteria (a 'female' disorder caused by their reproductive organs which makes women 'emotional' and 'disagreeable'), as well as the fact that homosexuality and even masturbation was considered a mental disorder (Bayer 1987; Ehrenreich and English 2013; see, also Gagné-Julien 2021). Better safeguarding against such misuses would be enabled by requiring that the criteria for application of the concept of dysfunction be thought of as generally independent from the criteria of application of the concept of harm. On the one hand, this enables us to protect the society against misuse of psychiatry such as witnessed in the case of drapetomania. In particular, cases such as 'drapetomania' in the nineteenth century, might be harmful because of unjust social structures and people's attitudes, but they should not be considered disorders because they are not underpinned by an underlying dysfunction. On the other hand, it enables us to protect against misuses as exhibited in the case of homosexuality. As argued before, this case teaches us that conditions, personality traits, mental states, and behaviors that are not intrinsically harmful should not be considered as disorders regardless of whether they are underpinned by some dysfunction. Granted, even today there are conditions whose underlying dysfunctionality cannot be unambiguously determined (see, e.g. Gagné-Julien 2021; Stegenga 2021). Such cases might leave us (implicitly or explicitly) heavily relying on harm and other socially-based evaluative considerations when trying to determine whether some trait is dysfunctional. This poses the similar danger of psychiatric misuse which the historical examples, such as the one of drapetomania, keep us wary of. However, it is our view that striving for the two criteria, one based on the individual's perspective in the form of harm, and one descriptive aspiring for scientific objectivity currently might be our best chance at minimizing the potential of psychiatric abuse. ${ }^{6}$

\footnotetext{
${ }^{6}$ And there is always a chance of getting things wrong. Sometimes we can become cognizant of things only in hindsight (for discussion of several recent case studies, see Gagné-Julien 2021; MurphyHollies 2021; Stegenga 2021). Thanks to an anonymous reviewer for pointing this out.
}

To sum up, we think that the requirements of theoretical and ethical fruitfulness favor keeping conceptual distinctions between the harm and dysfunction components. To be more precise, in comparison to the dysfunction-only views, we think they favor hybrid views of mental disorder as they enable more effective integration of scientific research across disciplines, while at the same time provide safety gates against potential misuses of psychiatry. In effect, such hybrid views empower the people affected by psychiatric practice to receive more opportunity and options for the recognition of their claims and needs.

\section{Conclusion}

In this paper, we have discussed some recent arguments for negating the importance of the notion of harm when defining the concept of mental disorder within DSM-5. The discussed criticisms are related to the fact that the concept of harm is currently underspecified in DSM-5 and, thus, not particularly useful for deciding in specific cases whether a person is mentally disordered.

For the purposes of evaluating these criticisms we have adopted the explicationist methodology. Based on this methodology we have argued that the concept of mental disorder should be explicated in terms of a hybrid view that construes mental disorders as harmful conditions that are underpinned by behavioral, psychological, or biological dysfunctions (whether they are construed as naturalistic or value-laden). We think that this account provides better grounds for delineating pathological from non-pathological conditions and more effectively secures from injustices that otherwise might be imposed by psychiatric practice.

Acknowledgements We would like to thank Luca Malatesti for reading and commenting on previous versions of this paper. Thanks also to two anonymous reviewers for Medicine, Health care, and Philosophy for very helpful helpful comments. Finally, we would like to thank the audiences at PSA 2020/2021 (Baltimore) and ZAEC 2021 (Zagreb) for their comments on previous versions of the paper. Mia Biturajac's work is supported by project "Responding to antisocial personalities in a democratic society" (RAD, Grant HRZZ-IP-2018-01-3518). Marko Jurjako's work is supported by project "Harm, intentions, and responsibility" (HIRe, HRZZ-UIP-2017-05-4308). Both projects are funded by the Croatian Science Foundation. Jurjako also thanks BIAS Institute (Nerezine) and University of Rijeka (Grant uniri-human-18-265) and University of Rijeka Small Grant for Internationalization of Research.

Funding Funding was provided by Hrvatska Zaklada za Znanost (DOK-2018-09-5165, HRZZ-IP-2018-01-3518, and HRZZUIP-2017-05-4308), University of Rijeka, Project KUBIM (uniri-human-18-265), and University of Rijeka (Small Grant for Internationalization). 


\section{References}

American Psychiatric Association. 1994. Diagnostic and statistical manual of mental disorders: DSM-IV, 4th ed. Washington, DC: American Psychiatric Association.

American Psychiatric Association. 2013. Diagnostic and statistical manual of mental disorders: DSM-5, 5th ed. Washington, DC: American Psychiatric Association.

Amoretti, M. Cristina, and Elisabetta Lalumera. 2019. Harm should not be a necessary criterion for mental disorder: Some reflections on the DSM-5 definition of mental disorder. Theoretical Medicine and Bioethics 40 (4): 321-337. https://doi.org/10.1007/ s11017-019-09499-4.

Amoretti, M. Cristina, and Elisabetta Lalumera. 2021. Wherein is the concept of disease normative? From weak normativity to valueconscious naturalism. Medicine, Health Care, and Philosophy. https://doi.org/10.1007/s11019-021-10048-x.

Bayer, Ronald. 1987. Homosexuality and American Psychiatry: The politics of diagnosis. Princeton, N.J: Princeton University Press.

Beebee, Helen, and Nigel Sabbarton-Leary. 2010. Are psychiatric kinds real? European Journal of Analytic Philosophy 6 (1): 11-27.

Bolton, Derek. 2008. What is mental disorder? An essay in philosophy, science, and values. Oxford: Oxford University Press.

Boorse, Christopher. 1975. On the distinction between disease and illness. Philosophy and Public Affairs 5 (1): 49-68.

Boorse, Christopher. 1977. Health as a theoretical concept. Philosophy of Science 44 (4): 542-573. https://doi.org/10.1086/288768.

Boorse, Christopher. 2014. A second rebuttal on health. The Journal of Medicine and Philosophy 39 (6): 683-724. https://doi.org/10. 1093/jmp/jhu035.

Brun, Georg. 2016. Explication as a method of conceptual re-engineering. Erkenntnis 81 (6): 1211-1241. https://doi.org/10.1007/ s10670-015-9791-5.

Carnap, Rudolf. 1971. Logical foundations of probability. 4. impr. Chicago: University of Chicago Press.

Carus, A.W. 2009. Carnap and twentieth-century thought: Explication as enlightenment. Cambridge: Cambridge University Press.

Cooper, Rachel V. 2002. Disease. Studies in History and Philosophy of Science Part C: Studies in History and Philosophy of Biological and Biomedical Sciences 33 (2): 263-282. https://doi.org/10.1016/ S0039-3681(02)00018-3.

Cooper, Rachel V. 2005. Classifying madness: A philosophical examination of the diagnostic and statistical manual of mental disorders. Dordrecht and New York: Springer.

Cooper, Rachel V. 2013. Avoiding false positives: Zones of rarity, the threshold problem, and the DSM clinical significance criterion. The Canadian Journal of Psychiatry 58 (11): 606-611. https:// doi.org/10.1177/070674371305801105.

Cooper, Rachel V. 2020. The concept of disorder revisited: Robustly value-laden despite change. Aristotelian Society Supplementary 94 (1): 141-161. https://doi.org/10.1093/arisup/akaa010.

Drescher, Jack. 2015. Out of DSM: Depathologizing homosexuality. Behavioral Sciences 5 (4): 565-575. https://doi.org/10.3390/ bs5040565.

Dutilh Novaes, Catarina. 2018. Carnapian explication and ameliorative analysis: A systematic comparison. Synthese. https://doi.org/10. 1007/s11229-018-1732-9.

Dutilh Novaes, Catarina, and Erich Reck. 2017. Carnapian explication, formalisms as cognitive tools, and the paradox of adequate formalization. Synthese 194 (1): 195-215. https://doi.org/10.1007/ s11229-015-0816-z.

Ehrenreich, Barbara, and Deirdre English. 2013. For Her own good: Two centuries of the experts advice to women. Knopf Doubleday Publishing Group.
Ereshefsky, Marc. 2009. Defining 'health' and 'disease.' Studies in History and Philosophy of Science Part C: Studies in History and Philosophy of Biological and Biomedical Sciences 40 (3): 221-227. https://doi.org/10.1016/j.shpsc.2009.06.005.

Fulford, K.W.M. 1989. Moral theory and medical practice. Cambridge: Cambridge University Press.

Gagné-Julien, Anne-Marie. 2021. Wrongful medicalization and epistemic injustice in psychiatry: The case of premenstrual dysphoric disorder. European Journal of Analytic Philosophy 17 (2): (SI4)536. https://doi.org/10.31820/ejap.17.3.3.

Garson, Justin, and Gualtiero Piccinini. 2013. Functions must be performed at appropriate rates in appropriate situations. The British Journal for the Philosophy of Science 65 (1): 1-20.

Glover, Jonathan. 1970. Responsibility. London: Humanities P.

Graham, George. 2013. The disordered mind: An introduction to philosophy of mind and mental illness, 2nd ed. Oxford: Routledge.

Griffiths, Paul E., and John Matthewson. 2018. Evolution, dysfunction, and disease: A reappraisal. The British Journal for the Philosophy of Science 69 (2): 301-327. https://doi.org/10.1093/ bjps/axw021.

Haslanger, Sally. 2012. Resisting reality: Social construction and social critique. Oxford: Oxford University Press.

Hausman, Daniel M. 2012. Health, naturalism, and functional efficiency. Philosophy of Science 79 (4): 519-541. https://doi.org/ $10.1086 / 668005$.

Hofmann, Bjørn. 2021. How to draw the line between health and disease? Start with suffering. Health Care Analysis 29 (2): 127-143. https://doi.org/10.1007/s10728-021-00434-0.

Jurjako, M., and L. Malatesti. 2020. In what sense are mental disorders brain disorders? Explicating the concept of mental disorder within RDoC. Phenomenology and Mind 18: 182-198.

Jurjako, M., L. Malatesti, and I.A. Brazil. 2021. The societal response to psychopathy in the community. International Journal of Offender Therapy and Comparative Criminology. https://doi.org/ 10.1177/0306624X211023918.

Kennedy, Ashley Graham, and Bryan Cwik. 2021. Diagnostic justice: Testing for Covid-19. European Journal of Analytic Philosophy 17 (2): (SI2)5-25. https://doi.org/10.31820/ejap.17.3.1.

Kingma, Elselijn. 2014. Naturalism about health and disease: Adding nuance for progress. The Journal of Medicine and Philosophy 39 (6): 590-608. https://doi.org/10.1093/jmp/jhu037.

Lancellotta, Eugenia, and Lisa Bortolotti. 2020. Delusions in the two-factor theory: Pathological or adaptive? European Journal of Analytic Philosophy 16 (2): 37-57. https://doi.org/10.31820/ ejap.16.2.2.

Lemoine, Maël. 2013. Defining disease beyond conceptual analysis: An analysis of conceptual analysis in philosophy of medicine. Theoretical Medicine and Bioethics 34 (4): 309-325. https://doi. org/10.1007/s11017-013-9261-5.

Matthewson, John, and Paul E. Griffiths. 2017. Biological criteria of disease: Four ways of going wrong. The Journal of Medicine and Philosophy: A Forum for Bioethics and Philosophy of Medicine 42 (4): 447-466. https://doi.org/10.1093/jmp/jhx004.

Murphy, Dominic. 2006. Psychiatry in the scientific image. Cambridge, MA: The MIT Press.

Murphy-Hollies, Kathleen. 2021. When a hybrid account of disorder is not enough: The case of gender dysphoria. European Journal of Analytic Philosophy 17 (2): (SI6)5-37. https://doi.org/10.31820/ ejap.17.3.5.

Nordenfelt, Lennart. 1995. On the nature of health: An action-theoretic approach. Berlin: Springer.

PhillipsR., Michael. 2009. Is distress a symptom of mental disorders, a marker of impairment, both or neither? World Psychiatry 8 (2): 91-92.

Powell, Russell, and Eric Scarffe. 2019. "Rethinking "disease": A fresh diagnosis and a new philosophical treatment.' Journal of 
Medical Ethics 45 (9): 579-588. https://doi.org/10.1136/medet hics-2019-105465.

Queloz, Matthieu. 2021. The practical origins of ideas: Genealogy as conceptual reverse-engineering. Oxford: Oxford University Press.

Rogers, Wendy A., and Mary Jean Walker. 2017. The line-drawing problem in disease definition. The Journal of Medicine and Philosophy 42 (4): 405-423. https://doi.org/10.1093/jmp/jhx010.

Schramme, Thomas. 2021. The quantitative problem for theories of dysfunction and disease. European Journal of Analytic Philosophy 17 (2): (SI7)5-29. https://doi.org/10.31820/ejap.17.3.6.

Schwartz, Peter H. 2007. Defining dysfunction: Natural selection, design, and drawing a line. Philosophy of Science 74 (3): 364385. https://doi.org/10.1086/521970.

Schwartz, Peter H. 2014. Reframing the disease debate and defending the biostatistical theory. The Journal of Medicine and Philosophy 39 (6): 572-589. https://doi.org/10.1093/jmp/jhu039.

Spitzer, R.L. 1973. A proposal about homosexuality and the APA nomenclature: Homosexuality as an irregular form of sexual behavior, and sexual orientation disturbance as a psychiatric disorder: A symposium. should homosexuality be in the APA nomenclature? American Journal of Psychiatry 130: 1207-1216.

Spitzer, R.L. 1981. The diagnostic status of homosexuality in DSM-III: A reformulation of the issues. The American Journal of Psychiatry 138 (2): 210-215. https://doi.org/10.1176/ajp.138.2.210.

Spitzer, R. L., and J. B. Williams. 1982. The definition and diagnosis of mental disorder. In Deviance and mental illness, edited by Walter R. Gove, 15-31. Sage Annual Reviews of Studies in Deviance, v. 6. Beverly Hills: Sage Publications.

Stegenga, Jacob. 2015. Effectiveness of medical interventions. Studies in History and Philosophy of Science Part C: Studies in History and Philosophy of Biological and Biomedical Sciences 54: 34-44. https://doi.org/10.1016/j.shpsc.2015.06.005.
Stegenga, Jacob. 2021. Medicalization of sexual desire. European Journal of Analytic Philosophy 17 (2): (SI5)5-32. https://doi. org/10.31820/ejap.17.3.4.

Telles-Correia, Diogo. 2018. Mental disorder: Are we moving away from distress and disability? Journal of Evaluation in Clinical Practice 24 (5): 973-977. https://doi.org/10.1111/jep.12871.

Varga, Somogy. 2017. Mental disorder between naturalism and normativism. Philosophy Compass 12 (6): e12422. https://doi.org/ 10.1111/phc3.12422.

Veit, Walter. 2021. Biological normativity: A new hope for naturalism? Medicine, Health Care and Philosophy. https://doi.org/10.1007/ s11019-020-09993-w.

Wakefield, Jerome C. 1992. The concept of mental disorder. On the boundary between biological facts and social values. The American Psychologist 47 (3): 373-388.

Wakefield, Jerome C. 2014. The biostatistical theory versus the harmful dysfunction analysis, Part 1: Is part-dysfunction a sufficient condition for medical disorder? Journal of Medicine and Philosophy 39 (6): 648-682. https://doi.org/10.1093/jmp/jhu038.

Walker, Mary Jean, and Wendy A. Rogers. 2018. A new approach to defining disease. The Journal of Medicine and Philosophy 43 (4): 402-420. https://doi.org/10.1093/jmp/jhy014.

World Health Organization. 1992. The ICD-10 Classification of mental and behavioural disorders: Clinical descriptions and diagnostic guidelines. Geneva: World Health Organization.

Publisher's Note Springer Nature remains neutral with regard to jurisdictional claims in published maps and institutional affiliations. 